Instituto Internacional de Investigación y Desarrollo Tecnológico Educativo INDTEC, C.A.

DOI: https://doi.org/10.29394/Scientific.issn.2542-2987.2018.3.8.7.138-158

OAI-PMH: http://www.indteca.com/ojs/index.php/Revista Scientific/oai

\title{
La Educación Sexual de la Mujer en Sectores Vulnerables
}

Autora: Lynett Carolina Vásquez Veracochea Universidad Fermín Toro, UFT

lybarbie@hotmail.com

Lara, Venezuela

\section{Resumen}

El presente artículo brinda una descripción de un trabajo multidisciplinario con el objetivo de proponer el desarrollo de estrategias orientadoras necesarias para informar y fomentar la educación sexual y reproductiva a las mujeres del Municipio Manuel Monge del estado Yaracuy, Venezuela; permitiendo un aporte activo a la sociedad para contrarrestar los embarazos adolescentes, pobreza extrema y la erradicación de la violencia de género, buscando el empoderamiento de la mujer en sectores vulnerables a partir del conocimiento de sus derechos. La metodología empleada desde la investigación fue la acción participación con un enfoque del paradigma cualitativo por la percepción de los actores, un diseño previo para la recolección de información, planificación de estrategias sobre conocimientos que permitiera elaborar actividades de entrevistas semiestructuradas a tres informantes claves de los grupos focales, con planes de trabajo, talleres prácticos, jornadas de salud y una escucha activa para establecer criterios de los recursos en la obtención de los resultados.

La técnica de interpretación de datos permite ordenar la información del cómo, dónde, cuándo y por qué con claridad a fin de establecer una relación entre categorías, y otros datos recolectados para brindar un acompañamiento integral a la mujer hacia el logro de los objetivos planteados. natalidad.

Palabras clave: educación sexual; planificación de la familia; tasa de 


\section{Sexual Education of Women in Vulnerable Sectors}

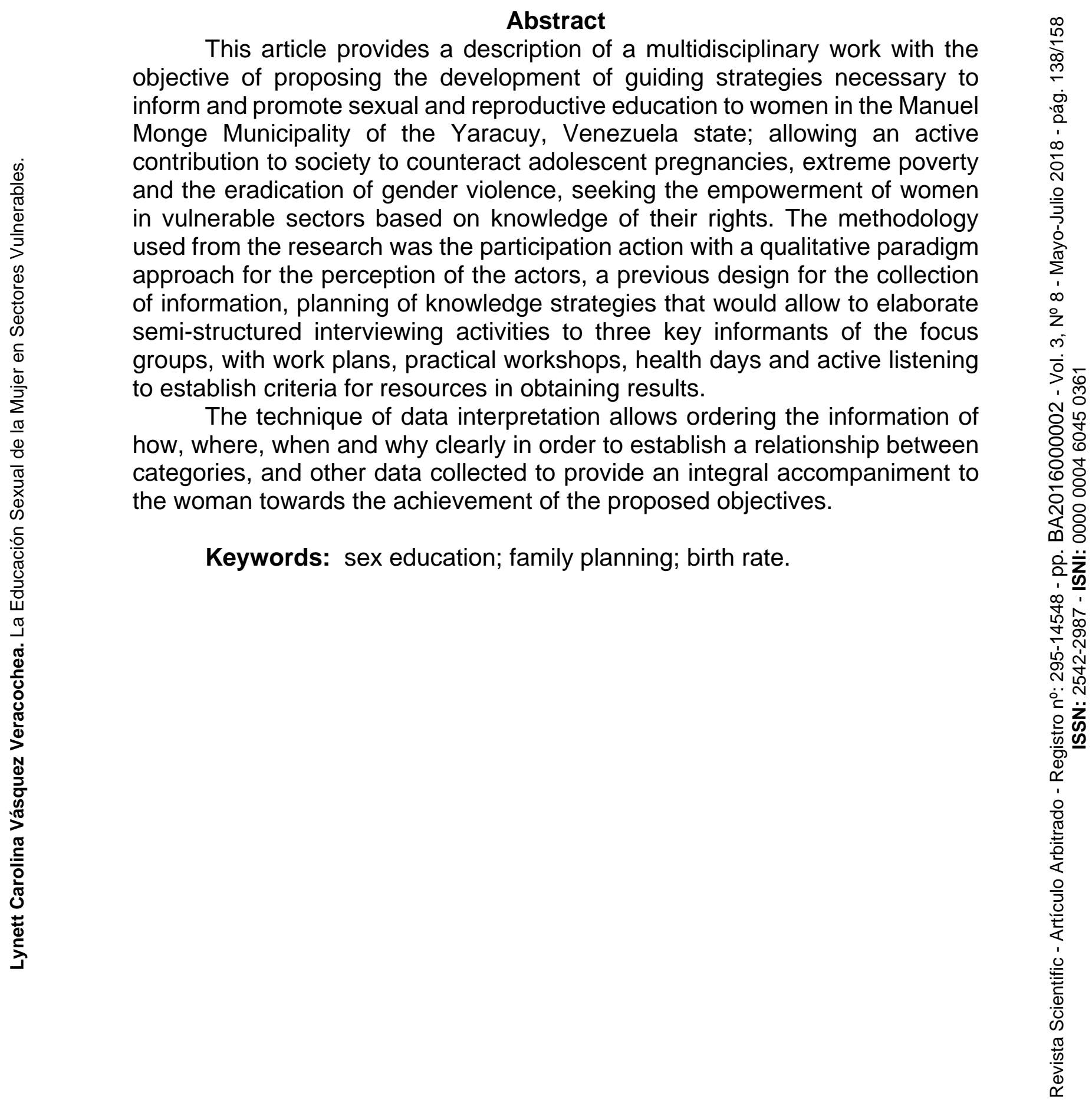

Date Received: 08-01-2018

Date Acceptance: 08-03-2018 


\section{Introducción}

Desde que el hombre comenzó a vivir en comunidad, implementó una serie de códigos que le permitieran compartir conciencia social, es decir un sentido compartido con la comunidad de su realidad; los inicios de la civilización en aspectos de convivencia parecen estar instaurados en un sistema de aparente igualdad en las relaciones sociales, de organización social y cultural. Desde su subjetividad las diferencias entre hombre mujer no establecía diferencia a la hora de afrontar cargos de trabajo y de poder.

Sin embargo, a medida que pasa el tiempo el poder, el conocimiento y nuevas dinámicas sociales, transformaron los significados grupales, la instauración de un modelo monoteísta partiendo de los judíos transforma el mundo dual de poder en el mundo androcéntrico, donde el sesgo que se instauró en el nuevo imaginario social sobre la información y formación de la mujer.

Con este sistema de conciencia social se garantizó en primer lugar el control sobre las conductas sociales, donde el sistema binario hombre-mujer tenía sus estereotipos culturales normativos limitando su participación dentro de los lineamientos establecidos desde relaciones de dominación. En estas lógicas socio simbólicas que reproduce y legitiman diferencias de poder como lo planteó Michael Foucault, las lógicas de poder necesitan de mecanismos de control y dominación que permitan legitimar sus prácticas.

El mayor avance a mediados del siglo XX, fue la creación de la pastilla anticonceptiva, que permitió a la mujer la reapropiación de su cuerpo, encontrarse nuevamente con una sexualidad castrada y negada, comenzando la verdadera independencia asumiendo la naturaleza hasta cierto punto castrada hasta ese momento. Sin embargo, el camino de la sexualidad como construcción social de las expresiones biológicas, emocionales y relacionales aún se encuentra influenciada por un contexto cultural que se sostiene sobre el patriarcado. 
Por su parte, el término de salud sexual ampliamente difundidos por la Organización Mundial de la Salud es definido como una construcción multifactorial del ser sexual donde intervienen aspectos intelectuales, culturales, sociales, emocionales y somáticos, vinculados a la capacidad de expresar la sexualidad de una manera libre y plena.

La educación sexual sigue siendo un tema tabú para las mujeres en la sociedad actual, desde el desenvolvimiento de la era cristiana; numerosos estereotipos difundidos a través de los medios han hecho que el ser social tenga conductas aprendidas y reforzadas desde la visión colectiva amparada en los agentes socializadores primarios; sobre este imaginario se cuenta con un sistema de medios occidental que ha retratado una realidad única y alienante capaz de unificar los criterios de belleza, verdad, bueno o malo.

Patrones religiosos, políticos, deportivos, culturales y sociales, son criticados con vehemencia por estos medios, cuando sostienen una posición diferente a la que se comparte desde las fuentes de poder dentro de cualquier estructura social del gobierno hasta las instituciones primarias como escuela y familia. Es por ello que la educación de la sexualidad se transforma en una responsabilidad de la sociedad en su conjunto, fomentada desde el nacimiento dentro del grupo familiar y transcurre durante toda la vida en los sucesivos vínculos que establecen las personas al interactuar con diferentes grupos.

El desarrollo de estos vínculos viene definido desde el factor ambiente, que genera características especiales dentro de la cultura que bien como señala (Durand, 2002, pág. 179): en su artículo sobre el impacto del ambiente en la cultura social: "El determinismo ambiental implica que la cultura está constituida básicamente por una serie de adaptaciones al ambiente y se desarrolla en un proceso equivalente al de la selección natural".

Con esta afirmación la autora aclara el papel indispensable del ambiente, como condicionador de posturas hacia determinados temas en este caso con lo referente a la educación sexual. Es por lo que para visualizar el 
caso que se abordará, se debe empezar por definir el ambiente donde se llevó a cabo este trabajo multidisciplinario ubicado en la zona agrícola rural del estado Yaracuy, colindante con el estado Falcón en Venezuela.

El Municipio bajo el nombre de Manuel Monge, posee una población de más de 30 mil habitantes, datos aportados desde el censo del Comité Local de Abastecimiento y Producción del ente gubernamental municipal donde cerca del $40 \%$ de esta, se encuentra en situación de pobreza a pobreza extrema caracterizándose la población por tener unos de los índices más altos de natalidad a nivel estadal y nacional en los últimos años especialmente en embarazos adolescentes según cifras extraoficiales de salud Municipal.

\section{Desarrollo}

\subsection{Reconocimiento de la Necesidad}

Dentro de las actividades realizadas a partir del Gobierno Municipal representada en la Alcaldía del Municipio Manuel Monge, uno de los 14 que conforman el estado Yaracuy en Venezuela, se encuentra una modalidad llamada gobierno de calle donde la máxima autoridad municipal se dirige a las 21 comunidades extendidas en sus $474 \mathrm{~km} 2$ con un equipo multidisciplinario buscando abordar las diferentes problemáticas que se presenten en sectores, emergiendo, varias necesidades más allá de los simplemente materiales.

En este caso, la visión se presenta desde la perspectiva comunicacional, psicológica y educativa, áreas de estudio del investigador social para desarrollar las herramientas que permitan llevar un mensaje que represente un aporte al desarrollo bio-psico social del individuo; el principal sujeto de abordaje resulta la mujer, debido a su posición transcendental dentro de los sectores vulnerables, principalmente por su capacidad de dar vida durante su edad reproductiva, aumentando la población en los sectores de escasos recursos.

Si bien, el abordaje de las relaciones sexuales y la reproducción radica 
en una perspectiva de corresponsabilidad de hombre mujer, la misma se encuentra en una posición de vulnerabilidad cultural y sexual debido a la sociedad patriarcal que le ha quitado derechos sobre su sexualidad con juicios de valor y etiquetas culturales que les llenan de inseguridad a la hora de buscar ayuda profesional al tomar la decisión de tener relaciones sexuales especialmente en los jóvenes; Calero (2007), citado por Rodríguez, Sanabria, Contreras y Perdomo (2013), afirma en su discurso que:

El grado de vulnerabilidad del adolescente y joven está en función de su historia anterior, sumada a la susceptibilidad o fortalezas inducidas por los cambios de algunos factores sociales, demográficos, el microambiente familiar, las políticas socioeconómicas, así como el grado de conocimiento que poseen sobre Salud Sexual y Reproductiva. (pág. 162).

\subsection{El Poder y la Sexualidad}

La sociedad está compuesta de varias estructuras de poder, conocida desde la sociología, como estructura social que opera desde lo simbólico en el ejercicio del poder, teniendo dos sentidos complementarios como lo son la estructura relacional y la institucional; ambas permiten describir las relaciones que vinculan a miembros en comunidades organizadas. Estas organizaciones buscan crear cultura cargada de valores etnográficos, geográficos de utilidad política y cultural.

En estas estructuras de poder se encuentran posiciones como el machismo que se encuentra como formas de opresión, siendo objetivas haciendo predecibles las conductas humanas en búsqueda del poder. Este poder como lo reseñaba Foucault (1976): se presenta a partir de estas estructuras sociales que ejercen el control sobre las relaciones e interacciones de los sujetos normando su conducta; esta se logra a través de la construcción de subjetividades donde la sexualidad juega un papel preponderante en el saber y verdad hacia la emancipación del ser:

El sexo es a un tiempo acceso a la vida de un cuerpo y a la vida 
de la especie. Es utilizado como matriz de las disciplinas y principal de las regulaciones... Pero también se convierte en tema de operaciones políticas, de intervenciones económicas mediante incitaciones o frenos a la procreación de campañas ideológicas de moralización o de responsabilización, se le hace valer como índice de fuerza de una sociedad revelando así tanto su energía política como su vigor biológico. (pág. 87).

La posición del autor, permite reflexionar sobre el uso de la sexualidad como ente de control dentro de la sociedad, en este caso hablando de sectores vulnerables se evidencia la poca atención que existe al aumento de la tasa de nacimientos en personas de pobreza extrema que repercutirá en otros flagelos sociales como enfermedades, desnutrición, muerte, delincuencia entre otros atenuantes que perjudican el desarrollo de estos sectores, con herramientas para vencer las circunstancias de pobreza extrema hacia su emancipación ideológica.

Al realizar la observación del Municipio a partir de la escucha activa, se evidenciada en los sectores de vulnerabilidad, la presencia de mujeres adolescentes o jóvenes con varios niños, apelando al aporte activo del Estado para poder contar con alimentación y beneficios sociales.

En estas estructuras son pocas las que culminan el ciclo escolar, por lo que acceder al conocimiento de manera investigativa es difícil para ellas, el conocimiento se acoge de una manera vivencial, a través del día a día y el intercambio de saberes con el discurso de sus conciudadanos. Este discurso viene validado generalmente por el hombre, quienes generalmente son las figuras de poder, quienes llevan el aporte económico a cambio del ceder el poder de decisión en temas de índole sexual, evitando u obviando la protección a las mujeres para evitar enfermedades y embarazos no planificados.

Así se entrelazan las relaciones del biopoder, en estos sectores amparados en estereotipos sociales que se reproducen en las mujeres 
avalados en el discurso machista de las instituciones y discurso social, el desconocimiento del aparato legal de protección con el que cuenta la mujer en la actualidad para garantizar su desenvolvimiento para una vida libre de violencia.

\subsection{Sistema de Salud vs Atención Social}

Es importante señalar, que el área de salud municipal se encuentra implementando políticas de asistencia integral, coordinando con las instituciones regionales y nacionales para brindar la atención oportuna a sus habitantes en áreas de prevención y cuidado de forma gratuita para todos los habitantes del Municipio.

Detectado el problema de información y orientación en las áreas de salud sexual, los médicos ejecutaron respuestas médicas de atención con métodos anticonceptivos y charlas de planificación familiar. Estos recursos si bien eran populares dentro de la población femenina, cumplían como paliativos a un problema que día a día sigue en aumento por la poca concientización de los derechos como mujeres, anteponiendo la reproducción sobre la autorrealización ejemplificada en la pirámide de Maslow donde todas las necesidades sean cubiertas.

La atención social, comprendida como un trabajo multidisciplinario y altamente pedagógico, que pretenda la autonomía del ser era un tema innovador en el municipio, por lo que hubo resistencia en los grupos de las esferas médicas para acoplar un programa donde la prioridad fuera la construcción colectiva de la mujer con las herramientas para el ejercicio de su sexualidad de una manera libre y segura, pasando desde la teoría al activismo, visitando a lo largo y ancho del municipio con una educación critica que como decía (Freire, 2004a, pág. 12): "No buscara transferir conocimientos sino crear posibilidades para su construcción o producción".

Es por ello, que después de varias reuniones entre la investigadora 
social quien fungía como comisionada de la Alcaldía para el sector salud y comunicación de la Alcaldía y el gremio médico representado en la Coordinación de salud Municipal, se logra integrar la visión de hacer de la educación sexual en las comunidades una manifestación de la experiencia vital con aportes históricos que serán construidos y deconstruidos desde lo colectivo hasta lograr la transformación dándole sentido a todo el aparato simbólico que rige la sexualidad en la actualidad en estas zonas de escasos recursos.

\subsection{Objetivo}

Es así, como se plantea el objetivo principal del trabajo multidisciplinario que será el desarrollo de estrategias orientadoras necesarias para informar y fomentar la educación sexual y reproductiva a las mujeres del Municipio Manuel Monge del estado Yaracuy, Venezuela a través de estrategias pedagógicas que permitan la transformación social que empodere a las mujeres especialmente en condiciones de vulnerabilidad económica, psicológica o social.

\subsection{Dialéctica del Ser y el Hacer}

Para iniciar la creación de las nuevas estrategias orientadoras pedagógicas, es necesario ejercer la práctica educativa con una actitud positiva como bien lo decía el mismo Freire, ya que, para cambiar al mundo con un ímpetu libre y necesario de que un mejor mundo con la esperanza de que el cambio es posible.

En este camino, es indispensable reforzar la escucha activa para los educandos que en este caso son las mujeres de extrema pobreza y poco o ningún acceso a la información sobre sus deberes y derechos como mujeres en este país. Para ello se sigue la recomendación del mismo Freire (2004b), de realizar un psicoanálisis histórico, político y social que permitan a las participantes reconocer el sistema excluyente deshumanizante que genera su 
situación de vulnerabilidad.

Los procesos pedagógicos, funcionan como creaciones incesantes y a partir de ellas se abre un camino de múltiples opciones para seguir avanzando replanteándose siempre los objetivos hacia una perfecta articulación entre la teoría y la praxis fortaleciendo la dialogicidad entre el ser y el hacer.

\section{Metodología}

\subsection{Elaboración del Taller}

La naturaleza de este trabajo posee un enfoque cualitativo al considerar que el estudio del caso anteriormente planteado se apoya en el descubrimiento de informaciones, datos aportados directamente de los participantes a través de sus discursos.

El enfoque cualitativo es sustentado con el discurso de (Hernández, Fernández y Baptista 1997), que afirmaban que:

Los estudios involucran la recolección de datos utilizando técnicas que no pretenden medir ni asociar las mediciones con números, tales como observaciones no estructuradas, entrevistas abiertas, revisión de documentos, discusión en grupo, evaluación de experiencias personales, inspección de historias de vida, análisis semánticos y de discursos cotidianos, interacción de grupos o comunidades e introspección. (pág. 93).

También es importante aclarar, que desde esta perspectiva se puede vislumbrar el discurso del investigador de este trabajo que posee una vinculación hacia el área de formación, es decir: Conociendo el proceso me conozco. Es así como el acto de interpretar no puede ser fundamentado en una técnica sobre la cual fundar metodológicamente las ciencias humanas; comprender será entonces el modo propio a través del cual el sujeto se relaciona con el mundo social y físico.

Es entonces, cómo se intentará develar los patrones de la educación 
sexual y reproductiva impuesta por la estructura social permitiendo la participación legítima, igualitaria y directa de todas las partes implicadas en la construcción de su significado contrarrestando el modelo positivista de dominación y control.

Para el desarrollo de la indagación, se seleccionó el método de la Investigación Acción Participante porque establece en sus procedimientos elementos que permiten la participación cooperativa de sus sujetos e investigadores apuntando a la producción de conocimientos transformadores desde un proceso de construcción colectiva de saberes produciendo la transformación social mediante todos los actores. Según (Martínez, 1997, pág. 73), afirma que: "La investigación participativa se caracteriza por un conjunto de principios, normas y procedimientos metodológicos, que permite obtener conocimientos colectivos sobre una determinada realidad social".

Las técnicas recomendadas por este investigador indican que las mismas se basan en la integración de los sujetos de investigación con los miembros de la comunidad que participan en la transformación social donde el diagnostico grupal permite se ejecuten acciones de manera cíclica en fases que permiten el diagnostico, planificación, ejecución y la evaluación o resultados.

Para elaborar un diagnóstico de la realidad social de las mujeres en el Municipio, en cuanto a la educación sexual con miras a crear estrategias orientadoras para su desarrollo, se procedió bajo la Acción participante se tomaron tres actores sociales quienes fungirán como nuestros informantes claves que fueron mujeres de la comunidad aportando datos relevantes para la planificación y ejecución del objetivo. Según Martínez (2002):

Un buen informante clave puede desempeñar una función decisiva en la investigación ya que introduce al investigador ante los demás, le sugiere ideas y formas de relacionarse, le previene de los peligros, responde por el ante el grupo y en resumen le hace puente con la comunidad. (pág. 54). 
En tal sentido, a los tres informantes claves se les aplicó una entrevista semiestructurada con la utilidad de recursos técnicos necesarios como cámara fotográfica, papel y lápiz, avistando a los informantes desde la cotidianidad de su entorno y así tomar su discurso desde la naturalidad. Apoyado en Martínez (2004): quien indica que la investigación acción participativa transformadora se establece dentro de la ciencia social critica que produce conocimiento reflexivo. Por tanto, las exploraciones educativas que siguen enfoques interpretativos y sociocríticos utilizan metodologías cualitativas que abordan el discurso de actores y autores de los contextos formativos.

Cuadro 1. Actividad: Registro de entrevista a informante clave 1.

\begin{tabular}{|c|c|c|c|}
\hline N.으 & Descripción & Subcategorías & Categorías \\
\hline 1 & $\begin{array}{l}\text { Presentación: } \\
\text { Me llamó Mayerlin Jiménez tengo } 26 \text { años } \\
\text { ama de casa con } 10 \text { hijos (un niño } \\
\text { prematuro de un mes) de la comunidad de } \\
\text { la 7; vivo con mis hijos, y una pareja no } \\
\text { estable que es el padre de mis últimos } \\
\text { cuatro hijos. El año pasado tuve un parto } \\
\text { de gemelos en el mes de septiembre los } \\
\text { cuales fallecieron al ser prematuros. } \\
\text { Deseo algún tipo de ayuda ya que no } \\
\text { cuento con recursos económicos. }\end{array}$ & $\begin{array}{l}\text { Planificación } \\
\text { Familiar } \\
\text { Pobreza } \\
\text { Embarazo } \\
\text { Adolescente } \\
\text { Métodos } \\
\text { anticonceptivos }\end{array}$ & $\begin{array}{l}\text { Estrategias } \\
\text { orientadoras } \\
\text { Educación } \\
\text { Sexual } \\
\text { Estrategias } \\
\text { pedagógicas }\end{array}$ \\
\hline
\end{tabular}

Fuente: La Autora (2017).

Cuadro 1.1. Actividad: Registro de entrevista a informante clave 1.

\begin{tabular}{|l|l|l|}
\hline ¿A qué edad tuviste tu primer hijo? & Valores & \\
A los 14 años, mi mamá me dejó en una & & \\
casa de familia y salí embarazada. & Planes de & \\
¿Alguna vez has recibido orientación & Atención Social & \\
sobre planificación familiar? & Integral & \\
Nunca he escuchado sobre el tema, no fui & & \\
a la escuela. & & \\
¿Qué apoyo se te ha dado por parte del & & \\
\hline
\end{tabular}




\begin{tabular}{|l|l|l|}
\hline estado? & \\
Apoyo de dinero y comida para cuidar a & \\
mis hijos. & ¿Cuáles son las necesidades que vez \\
con más frecuencia en las mujeres de tu & \\
sector? & \\
Aquí existe mucha pobreza, el dinero no & \\
alcanza, necesitan ayuda con sus niños. & \\
\hline
\end{tabular}

Fuente: La Autora (2017).

Cuadro 2. Actividad: Registro de entrevista informante clave 2.

\begin{tabular}{|c|c|c|c|}
\hline N.은 & Descripción & Subcategorías & Categorías \\
\hline 2 & $\begin{array}{l}\text { Descripción: Maribel Tovar } \\
\text { Tengo } 30 \text { años y soy de la comunidad de } \\
\text { la } 8 \text {, con tres hijos, de los cuales dos niños } \\
\text { son especiales estoy interesada en que el } \\
\text { estado me ayude a conseguir recursos } \\
\text { para ayudarme a cuidar a mis hijos y no } \\
\text { tener más niños. } \\
\text { ¿A qué edad tuviste tu primer hijo? } \\
\text { A los } 17 \text {, es una niña ella es muy inteligente } \\
\text { va a la escuela, quiere ser médico. Mis } \\
\text { otros dos niños tienen } 6 \text { y cuatro años uno } \\
\text { fue diagnosticado con autismo y el otro aun } \\
\text { no me dan diagnóstico. } \\
\text { ¿Alguna vez has recibido orientación } \\
\text { sobre planificación familiar? } \\
\text { Si, sé que existe quisiera que el estado me } \\
\text { ayudara ya que no puedo tener más niños } \\
\text { pues los costos con dos niños especiales } \\
\text { son muy grandes. }\end{array}$ & $\begin{array}{l}\text { Planificación } \\
\text { Familiar } \\
\text { Pobreza } \\
\text { Embarazo } \\
\text { Adolescente } \\
\text { Programas de } \\
\text { Salud } \\
\text { Métodos } \\
\text { anticonceptivos } \\
\text { Valores } \\
\text { Planes de } \\
\text { Atención Social } \\
\text { Integral }\end{array}$ & $\begin{array}{l}\text { Estrategias } \\
\text { orientadoras } \\
\text { Educación } \\
\text { Sexual } \\
\text { Estrategias } \\
\text { pedagógicas }\end{array}$ \\
\hline
\end{tabular}

Fuente: La Autora (2017).

Cuadro 2.1. Actividad: Registro de entrevista informante clave 2.

\begin{tabular}{|l|l|l|l|}
\hline $\begin{array}{l}\text { ¿Qué apoyo se te ha dado por parte del } \\
\text { estado? }\end{array}$ & $\begin{array}{l}\text { Jornadas de } \\
\text { esterilización }\end{array}$ & $\begin{array}{l}\text { Estrategias } \\
\text { orientadoras } \\
\text { menores no van al colegio. }\end{array}$ & $\begin{array}{l}\text { Educación } \\
\text { Sexual }\end{array}$ \\
\hline
\end{tabular}




\begin{tabular}{|l|l|l|l|}
\hline $\begin{array}{l}\text { ¿Cuáles son las necesidades que vez } \\
\text { con más frecuencia en las mujeres de tu } \\
\text { sector? }\end{array}$ & género \\
$\begin{array}{l}\text { Necesitan apoyo para cuidarse de los } \\
\text { hombres, las gritan o les pegan, igual hay } \\
\text { muchas que son violentas con los niños. }\end{array}$ & Autoestima & $\begin{array}{l}\text { Estrategias } \\
\text { pedagógicas }\end{array}$ \\
\hline
\end{tabular}

Fuente: La Autora (2017).

Cuadro 3. Actividad: Registro de entrevista informante clave 3.

\begin{tabular}{|c|c|c|c|}
\hline NN.ㅇ & Descripción & Subcategorías & Categorías \\
\hline 3 & $\begin{array}{l}\text { Descripción: Milka Torres soy del sector } \\
\text { de la } 22 \text { con } 35 \text { años } 5 \text { hijos: } 19,18,15,12 \\
\text { años y mes y medio, Ama de casa solicito } \\
\text { apoyo del estado para protegerme. } \\
\text { ¿A qué edad tuviste tu primer hijo? } \\
\text { A los } 16 \text { de mi primer novio, ambos } \\
\text { asistíamos a la escuela, él es mi pareja } \\
\text { desde hace } 20 \text { años. } \\
\text { ¿Alguna vez has recibido orientación } \\
\text { sobre planificación familiar? } \\
\text { En el colegio la recibí, sé que existen } \\
\text { métodos, pero son costosos. } \\
\text { ¿Qué apoyo se te ha dado por parte del } \\
\text { estado? } \\
\text { Pues en comida y salud también con la } \\
\text { educación de mis hijos. } \\
\text { ¿Cuáles son las necesidades que vez } \\
\text { con más frecuencia en las mujeres de tu } \\
\text { sector? } \\
\text { Aquí existen muchas niñas embarazadas, } \\
\text { ojalá e hicieran ayudas para ellas para qué } \\
\text { se cuidaran. }\end{array}$ & $\begin{array}{l}\text { Planificación } \\
\text { Familiar } \\
\text { Pobreza } \\
\text { Embarazo } \\
\text { Adolescente } \\
\text { Programas de } \\
\text { Salud } \\
\text { Métodos } \\
\text { anticonceptivos } \\
\text { Valores } \\
\text { Planes de } \\
\text { Atención Social } \\
\text { Integral } \\
\text { Derechos } \\
\text { Sexuales y } \\
\text { Reproductivos }\end{array}$ & $\begin{array}{l}\begin{array}{l}\text { Estrategias } \\
\text { orientadoras }\end{array} \\
\text { Educación } \\
\text { Sexual } \\
\text { Estrategias } \\
\text { pedagógicas }\end{array}$ \\
\hline
\end{tabular}

Fuente: La Autora (2017). 
Cuadro 4. Categorías y Subcategorías.

\begin{tabular}{|l|l|}
\hline \multicolumn{1}{|c|}{ Categorías } & \multicolumn{1}{c|}{ Subcategorías } \\
\hline \multirow{2}{*}{ Educación Sexual } & Embarazo Adolescente. \\
& Métodos anticonceptivos. \\
& Jornadas de esterilización. \\
& Derechos Sexuales y Reproductivos. \\
\hline \multirow{3}{*}{ Estrategias Orientadoras } & $\begin{array}{l}\text { Planificación Familiar. } \\
\text { Pobreza. }\end{array}$ \\
& Violencia de género. \\
& Autoestima. \\
\hline \multirow{3}{*}{ Estrategias pedagógicas } & Valores. \\
& Planes de Atención Social Integral. \\
& Talleres. \\
& Jornadas. \\
& Asesorías. \\
& Campañas de Concientización. \\
\hline
\end{tabular}

Fuente: La Autora (2017).

Con esta identificación de categorías, obtenidas en este primer instrumento, se procedió a la elaboración de un plan de acción iniciando con la elaboración de un taller de concientización sobre la sexualidad; para ello se elaboraron estrategias de articulación con diferentes dependencias, sin embargo la única que recibió respuesta en esta primera fase fue el organismo municipal del SEPNA (servicio de protección al niño y adolescentes) donde la psicóloga social de esta institución se incorporó a la idea propuesta cubriendo las áreas de autoestima, sexualidad, identidad, y perspectivas de género.

\subsection{Contenido del Taller}

El taller se tituló Mujer de Hoy, este nombre surgió por la justificación dada anteriormente sobre el papel de la mujer en la sociedad actual y su necesidad de empoderamiento para lograr superar las adversidades. Se abordó la introducción por la líder de la Comunidad para los presentes, sobre el programa que iniciaba ese día, allí la psicóloga institucional comenzó 
abordando el concepto de autoestima, de la niñez y los adultos, así como las perspectivas de la sexualidad, genero, identidad sexual y sexo en general, estableció una visión sobre el maltrato como representación de la baja autoestima y sus posibles consecuencias en el desarrollo del niño.

La segunda fase del taller estuvo a cargo de la investigadora social, se habló de la perspectiva de la mujer dentro de la sociedad, mediante la participación se buscó reflexionar en un debate los imaginarios actuales donde solo definen como madre dejando de lado la imagen sensual sin procreación, así como métodos de protección a la violencia en cualquiera de sus formas.

Se trata de sacar al opresor dentro del oprimido, buscando la respuesta desde la visión subjetiva y autónoma de cada una de ellas, logrando un aporte colectivo hacia lo que es ser mujer, las relaciones sexuales, los tabúes, la censura al ejercer una sexualidad responsable y las posibles consecuencias en sectores machistas al asumir posiciones críticas sobre la autodeterminación, sobre los derechos sexuales y reproductivos, buscando fortalecer la identidad de las mujeres que muchas veces son oprimidas buscando el empoderamiento de las mismas en su vida familiar.

En este tema, se tocó los avances de la Organización de las Naciones Unidas desde 1975, en la protección de los derechos de la Mujer, especialmente cuando se encuentran en situaciones vulnerables como pobreza o dominación económica, sexual y reproductiva por su pareja. Allí también se abordó el marco legal en Venezuela con la Ley Orgánica sobre el derecho de las Mujeres a una vida libre de Violencia (2007): que contempla un cambio de los estigmas sociales para con la mujer, estableciendo en su Artículo 1 que estará: "impulsando cambios en los patrones socioculturales que sostienen la desigualdad de género y las relaciones de poder sobre las mujeres, para favorecer la construcción de una sociedad justa democrática, participativa, paritaria y protagónica".

Al igual se habló de la responsabilidad y corresponsabilidad al asumir 
los riesgos y consecuencias de las relaciones sexuales, finalizando con la necesidad de empoderarlas dándoles el mensaje de informarse con las personas capacitadas, comunicarse con quienes puedan ayudarlas, protegerse de enfermedades y embarazos seguidos dejando al final el mensaje motivador que son ellas quienes deciden que planes hacer para su vida.

\subsection{Análisis de los Resultados}

Esta serie de talleres que recorrieron el Municipio, abarcaron más de cuatro comunidades, iniciando en la población de la 13, siguiendo por la capital del Municipio Yumare, en escuelas de Tesorero y el sector de la cero llevando las herramientas a más de 200 mujeres en edad reproductiva autogestionándose con implementos que hicieran más vistosas las actividades como el uso del video beam, las láminas, la computadora y la realización de

unos recuerdos para las asistentes sobre los derechos sexuales y reproductivos.

Para la elaboración del uso de las estrategias didácticas, se tomaron en cuenta la pertinencia, eficiencia y eficacia para las mejoras de la calidad educativa fundamentadas por Terán, Perdomo y Peña (2017): apuntan que: "La calidad debe estar conjugada con la pertinencia y el impacto en un contexto real global y multidimensional pues no se puede concebir una institución educativa de calidad que no sea pertinente con su entorno social”. (pág. 45).

En este punto se toma la institución educativa desde el contexto de lo simbólico, del acto de educar y aprender. Durante las entrevistas, para las declaraciones de prensa se expresaron de manera positiva de la actividad con que inició el gobierno de calle, recomendaron traer una segunda fase para toda la comunidad con más información, invitando a recorrer el municipio con este taller integrador y educativo. 


\section{Conclusiones y Recomendaciones}

Luego del interés de la colectividad y los alcances experimentados desde la primera experiencia, con esta estrategia orientadora y pedagógica para fortalecer la educación sexual en las mujeres de pobreza extrema del municipio se logró tener un alcance en más de 200 mujeres que permitirán ser multiplicadores de la información vivencial recibida desde las experiencias socializadoras.

Se recomienda la incorporación de estas actividades dentro de los gobiernos de calle de la Alcaldía, o a escala nacional e internacional en vista de que es un aporte para resolver desde una perspectiva educativa los problemas de pobreza, planificación familiar, embarazos adolescentes y otros factores que se generan dentro de estos ambientes donde existen barreras de comunicación sobre la sexualidad y sus consecuencias.

El interés de los habitantes por las charlas, llevó a mejorar la experiencia con un equipo multidisciplinario con la participación de los médicos con las charlas sobre los métodos anticonceptivos que se ofrecen dentro de la salud pública para la protección y prevención de embarazos y enfermedades sexuales así como la activación de diferentes jornadas quirúrgicas y de colocación de implantes anticonceptivos para las interesadas motivadas a ejercer una sexualidad responsable a partir de los talleres.

Del mismo modo se preparó talleres para escuelas, abordando también niños, niñas y adolescentes para el cocimiento y ejercicio de sus derechos y como protegerse desde una perspectiva jovial y juvenil abordando temas de actualidad como la violencia escolar y sus implicaciones dentro de la conducta socio afectiva y sexual del niño y adolescente.

Mediante la participación, enmarcada en la corriente humanista se obtuvo pertinencia en cada una de las herramientas utilizadas para fomentar valores como el amor, la responsabilidad y el aporte individual del desarrollo de la sociedad como un todo, fundamentado en el pensamiento educativo liberador 
de Freire (2004c): a partir de la ética, el respeto a la dignidad y la propia autonomía.

Para concluir, se puede afirmar que este programa se encuentra instaurado desde una perspectiva humanista, siendo un elemento novedoso que puede promocionarse a nivel regional y nacional, atendiendo las necesidades de conocimiento que nos permitan crecer como sociedad y como país pues como lo dijo el Libertador Simón Bolívar "Moral y luces son nuestras primeras necesidades".

\section{Referencias}

Durand, L. (2002). La relación ambiente-cultura en antropología: recuento y perspectivas. Distrito Federal, México: Editorial Nueva Antropología, XVIII (61). Recuperado de:

http://www.redalyc.org/articulo.oa?id=15906109

Freire, P. (2004a,b,c). Pedagogía de la Autonomía. Sao Pablo, Brasil: Editorial Paz y Tierra.

Foucault, M. (1976). Historia de la Sexualidad. Tomo I, Madrid, España: Editorial Siglo XXI.

Ley Orgánica sobre el derecho de las Mujeres a una vida libre de Violencia (2007). Gaceta Oficial $N^{\circ}$ 38668, 23 de abril. Caracas, Venezuela: La Asamblea Nacional de la República Bolivariana de Venezuela. Recuperado de:

http://www.derechos.org.ve/pw/wp-content/uploads/11.-Ley-

Org\%C3\%A1nica-sobre-el-Derecho-de-las-Mujeres-a-una-Vida-Librede-Violencia.pdf

Hernández, R., Fernández, C., \& Baptista, P. (2003). Metodología de la Investigación. Tercera edición. México: Editorial McGraw-Hill.

Martínez, M. (2004). Ciencia y Arte de la metodología cualitativa. México: Editorial Trillas. 
Martínez, M. (2002). La Investigación Cualitativa Etnográfica en Educación. México: Editorial McGraw-Hill.

Martínez, M. (1997). El paradigma emergente: hacia una nueva teoría de la racionalidad científica. $2 a$ ed. México: Trillas.

Rodríguez, A., Sanabria, G., Contreras, M., \& Perdomo, B. (2013). Estrategia educativa sobre promoción en salud sexual y reproductiva para adolescentes y jóvenes universitarios. La Habana, Cuba: Revista Cubana de Salud Pública, 39(1), 161-174. Recuperado de: https://www.scielosp.org/pdf/rcsp/v39n1/spu15113.pdf

Terán, C., Perdomo, E., \& Peña, Y. (2017). Alternativas Didácticas de Lectura para el Fortalecimiento de la Calidad Educativa. Revista Scientific, 2(6), 29-51. Recuperado de:

https://doi.org/10.29394/scientific.issn.2542-2987.2017.2.6.2.29-51 


\section{Lynett Carolina Vásquez Veracochea}

e-mail: lybarbie@hotmail.com

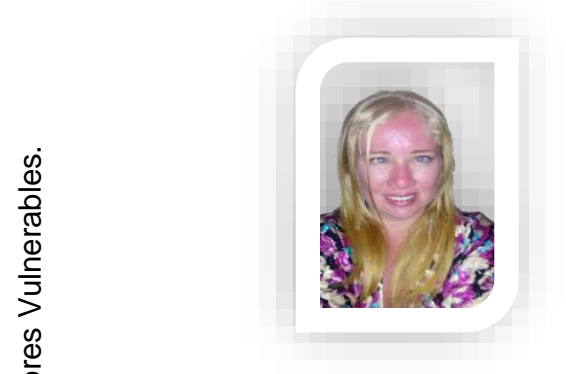

Nacida en Barquisimeto estado Lara, Venezuela.

Licenciada en Comunicación Social mención Periodismo de la UFT, Diplomado en gerencia de la Comunicación en la UCLA con una especialización en trabajo social de la UDO en Santiago de Cuba. Magister en Psicología Social mención análisis institucional y de grupos en la UARCIS de Santiago de Chile. Doctorante en Ciencias de la Educación, ha trabajado como Coordinadora de información y relaciones interinstitucionales en la UFT, Docente de la UPEL, escritora, productora de programas de asistencia social en salud y comunicación. Comisionada del despacho del Alcalde de Manuel Monge. Jefa de creación intelectual en la UNES.

El contenido de este manuscrito se difunde bajo una Licencia de Creative Commons ReconocimientoNoComercial-Compartirlgual 4.0 Internacional 